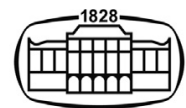

AKADÉMIAI KIADÓ

Journal of Psychedelic Studies

5 (2021) 2, 94-102

DOl:

10.1556/2054.2021.00179

(c) 2021 The Author(s)

ORIGINAL RESEARCH PAPER

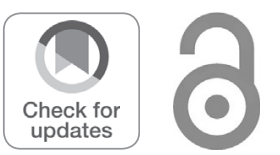

\section{Ketamine-assisted psychotherapy for trauma-exposed patients in an outpatient setting: A clinical chart review study}

\author{
ALAN K. DAVIS ${ }^{1,2 *}$ (D), PRATHEEK MANGINI ${ }^{3}$ and \\ YITONG XIN $^{1}$ \\ ${ }^{1}$ College of Social Work, Ohio State University, Columbus, OH, USA \\ ${ }^{2}$ Center for Psychedelic and Consciousness Research, Johns Hopkins University, Baltimore, MD, \\ USA \\ ${ }^{3}$ Department of Psychiatry, Rutgers-Robert Wood Johnson Medical School, New Brunswick, NJ, \\ USA
}

Received: May 3, 2021 • Revised manuscript received: September 8, 2021 • Accepted: September 15, 2021

Published online: October 1, 2021

\begin{abstract}
Trauma exposure across the lifespan produces risks for posttraumatic stress disorder (PTSD), depression, anxiety, as well as global disability in functioning. This retrospective clinical chart review is the first of its kind to assess the utility of sublingual ketamine-assisted body-centered psychotherapy in trauma-exposed patients in a real world clinic setting. De-identified clinical records data on self-reported symptom measures were retrospectively analyzed for patients $\left(N=18 ; M_{\text {age }}=45.22, S D=\right.$ 12.90) entering ketamine-assisted psychotherapy treatment in an outpatient clinic between 2018 and 2020. Patients who completed six sessions of ketamine therapy reported meaningful (e.g., medium effect size) improvements in PTSD symptoms $(P=0.058 ; d=-0.48)$ and global disability in functioning $(P=0.050 ; d=-0.52)$ and statistically significant and meaningful improvements in depression $(P=$ $0.019 ; d=-0.53)$. There were no improvements in anxiety symptoms. Sublingual ketamine-assisted psychotherapy was associated with heterogenous clinical utility among patients with trauma-exposure in an outpatient setting. This study was underpowered and unrepresentative of the population of ketamine patients in the United States. Replication of these findings is needed with larger and more diverse patient samples.
\end{abstract}

\section{KEYWORDS}

trauma, PTSD, depression, ketamine, mindfulness, psychotherapy

\section{INTRODUCTION}

Traumatic stress is widespread and often begins as early as childhood, wherein approximately one-half (45\%) of children experience stress secondary to at least one adverse childhood experience (ACE) (Crouch et al., 2019). Among adults, 70\% of individuals have been exposed to traumatic events, including war, physical and sexual assault, and traumatic deaths of loved ones (Koenen et al., 2017). High rates of trauma exposure have led to significant impacts on societal mental health, with $6 \%$ of people in the United States (US) reporting a lifetime prevalence of post-traumatic stress disorder (PTSD) (Koenen et al., 2017). Patients who have PTSD also experience high comorbidities for major depressive disorder (MDD), anxiety disorders, and substance use disorders (SUD) (Chilcoat \& Breslau, 1998; Kessler et al., 1995; Milanak et al., 2013; Pietrzak et al., 2012). Independent of a PTSD diagnosis, ACEs are correlated to the development of MDD, panic disorder, binge drinking, and increased suicide risk (Allem et al., 2015; Hughes et al., 2019; Maercker et al., 2004; Stein et al., 1996). In addition, trauma exposure has been associated with the development of insomnia, sleep
${ }^{*}$ Corresponding author. Tel.: +1614 2925251.

E-mail: davis.5996@osu.edu 
disorders, and daytime fatigue (Bader et al., 2007; Hall Brown, Akeeb, \& Mellman, 2015; Maher, Rego, \& Asnis, 2006; Neylan et al., 1998). PTSD patients also exhibit extreme dysfunction in family life and social impairment, reporting higher rates of divorce, struggles with parenting, and difficulties with emotional intimacy (Rodriguez, Holowka, \& Marx, 2012; Westphal et al., 2011). Impacts on work and academic performance have also been reported among patients with PTSD, wherein they have lost an average of 3.6 days of productivity per month (Kessler, 2000).

There are a variety of recommended therapies for PTSD treatment, including prolonged exposure (PE), eye movement desensitization reprocessing (EMDR), cognitive processing therapy, and trauma-focused cognitive-behavioral therapy (TF-CBT) (Watkins, Sprang, \& Rothbaum, 2018). Despite the numerous psychotherapeutic tools available to treat PTSD, there are several limitations of these interventions. For example, studies have found these treatment interventions' non-response rates ranging from $25 \%$ to $50 \%$ (Brady et al., 2015). Predictors of inadequate response correlated with perseverative thoughts, poor therapeutic alliance, and paucity of expression of thoughts and feelings (Brady et al., 2015). The difficulty in managing symptoms through psychotherapy is also compounded by high dropout rates, up to $48 \%$ (Imel et al., 2013).

Novel psychotherapeutic approaches include body-based mindfulness interventions to treat chronic, nonresponsive PTSD (van der Kolk et al., 2014). In one study (van der Kolk et al., 2014), 52\% of the participants in the intervention group no longer met the criteria for PTSD, compared to $21 \%$ in the control group. The authors hypothesized somatic regulation via "body awareness" facilitated improvement in symptoms (van der Kolk et al., 2014). Somatic experiencing, developed by Dr. Peter Levine, employs similar principles in a body-focused therapy. Through somatic experiencing therapy, an individual learns to tolerate distressing bodily sensations and associated emotions, allowing for the release and dissolution of stress activation. This approach differs from traditional exposure therapy as it focuses on selfregulation of arousal response by concentrating on somatic sensations instead of retelling traumatic narrative. A randomized controlled outcome study (Brom et al., 2017) found that participants $(N=63)$ assigned to somatic experiencing compared to those in a waitlist control had significant improvements in the severity of PTSD (Cohen's $d=0.94-1.26$ ) and depression symptoms (Cohen's $d=0.70-1.08$ ).

With respect to medication, two FDA-approved medications for treating PTSD are paroxetine and sertraline, both of which are selective-serotonin reuptake inhibitors (SSRIs). While sertraline had a $40 \%$ response rate among veterans (Brady et al., 2000; Panahi et al., 2011), one major limitation is that symptom relief requires 6-8 weeks with typical antidepressants. The delayed-onset efficacy is complicated by discontinuation syndromes, where sudden cessation can worsen PTSD symptoms and lead to increases in anxiety, depression, insomnia, and irritability (Warner et al., 2013). Furthermore, the evidence supporting sertraline is mixed with some studies showing no benefit and greater discontinuation rates $(30 \%)$ compared to placebo (17\%), hypothesized to be secondary to unreported adverse effects (Friedman et al., 2007).

Given these limitations, novel therapeutics, such as MDMA (Mitchell et al., 2021) and ketamine (Feder et al., 2014), are being assessed for their efficacy in PTSD with promising results. For example, recent Phase III trials of MDMA for PTSD are showing significant reductions in PTSD symptoms after treatment (Mitchell et al., 2021). Similarly, one study found that giving intraoperative ketamine to US army burn victims resulted in significant reductions in the prevalence of PTSD (McGhee et al., 2008). Besides, a preliminary trial compared intravenous ketamine to midazolam in patients with PTSD and found ketamine produced significantly rapid and pronounced reduction in PTSD symptom severity $24 \mathrm{~h}$ after infusion and comorbid depressive symptoms compared to midazolam (Feder et al., 2014). In addition, a case report of a 23 -year-old veteran diagnosed with severe chronic PTSD and depression from combat trauma was found to have a rapid, albeit temporary, reduction in PTSD symptoms following a single dose of IV ketamine (D'Andrea \& Andrew Sewell, 2013). The effects were sustained for 15 days before rapidly reversing (D'Andrea \& Andrew Sewell, 2013). Additional evidence supporting this treatment approach comes from a randomized controlled trial (Feder et al., 2021) comparing the efficacy of ketamine infusions to midazolam for chronic PTSD. After six infusions, the group that received ketamine demonstrated a stronger reduction in PTSD symptoms compared to the midazolam group (Feder et al., 2021). While there is a need for more research detailing the efficacy of ketamine in PTSD, the preliminary literature supports its potential as a treatment among trauma-exposed patients.

The safety and efficacy of ketamine have also been studied in anxiety disorders, particularly patients with refractory generalized anxiety disorder (GAD), social anxiety disorder (SAD), or both. Patients were given three ascending IV ketamine dose levels and midazolam at 1-week intervals. Ketamine dose-dependently improved symptoms by about $50 \%$ within one hour of dose with effects lasting up to 7 days after dosing (Shadli et al., 2018). A similar study evaluating maintenance treatment of IV ketamine in patients with $\mathrm{GAD}, \mathrm{SAD}$, or both $(N=20)$ found that patients had significant improvements in work functioning and reduced social avoidance (Glue et al., 2018).

With regards to ketamine's utility in depression, a review of 35 randomized controlled trials (RCTs) evaluating the benefits of ketamine for MDD found that intravenous ketamine was an effective treatment in approximately two-thirds of the trials (70\%; Memon et al., 2020). While most studies evaluated the role of IV ketamine, a systematic review assessing the utility of oral ketamine for depression across 13 studies (Rosenblat et al., 2019). Findings from this review showed that oral dosing of ketamine is tolerable with limited adverse effects. Remarkably, oral ketamine was able to produce medium to large effect sizes on anti-depressant effects but had a lag time of 2-6 weeks until the benefits were reported. 
Although there is evidence that ketamine is useful in PTSD, anxiety, and depressive disorders through IV or oral formulations, there is limited evidence on whether therapy could augment these benefits. One study evaluating ketamine-assisted psychotherapy (KAP) gave patients $(N=235)$ ketamine via sublingual (SL), intramuscular (IM), or both routes of administration in an outpatient setting. The patients had a variety of conditions, including depression, PTSD, and anxiety. The study found clinically significant improvements in depression scores decreased on average 11 points from moderate to mild depression. In addition, anxiety scores decreased on average 5 points from moderate to mild anxiety. Notably, patients with severe symptom burden at intake, such as those with higher scores, suicidality in the past year, history of psychiatric hospitalization, or higher ACE scores, had the most significant improvements. The authors postulate that KAP may produce altered mental states that open the patient to more reflective and vulnerable states, ultimately facilitating therapeutic rapport and progress (Dore et al., 2019).

Despite recent evidence suggesting potential clinical utility from ketamine-assisted therapy using sublingual and intramuscular ketamine (Dore et al., 2019), there has been no published report of the use of sublingual ketamine-assisted somatic psychotherapy for people with trauma-based disorders in a real world clinic setting. The present retrospective clinical chart review study is the first of its kind to assess whether the administration of sublingual ketamine in the context of bodycentered psychotherapy can provide benefits across several domains, including PTSD symptoms, depression, anxiety, and disability in functioning among patients with a history of trauma-exposure receiving treatment in an outpatient setting.

\section{METHODS}

\section{Clinical program}

Patients enrolled in treatment at an outpatient clinic in Colorado and underwent body-centered therapy augmented by sublingual ketamine administration. As part of standard clinical practice, patients completed a comprehensive battery of self-reported questionnaires at intake, and similarly completed symptom measures after six sessions depending on how long they were enrolled in treatment. In consultation with their therapist and a prescribing physician, patients were prescribed ketamine during their therapy sessions. These patients were prescribed sublingual lozenges of ketamine by a physician at doses typically ranging from 100 to $200 \mathrm{mg}$ approximately $10 \mathrm{~min}$ before therapy began. Patient were instructed to hold the lozenge under the tongue until it dissolved completely or as long as possible. Therapy sessions lasted approximately $120 \mathrm{~min}$, were typically facilitated by 1 or 2 therapists, and utilized a somatic-based interactional psychotherapy. This type of therapeutic approach is an autonomic nervous system-based body modality, which focuses on sensation, emotion, imagery, and nervous system reactivity. This treatment is designed to 1) focus on relational and personal aspects of a patient's psychological functioning, 2) activate the psychobiological autonomic nervous system to process psychological and physical experiences, and 3) establish a physical processing pathway to assist with resolution of nervous system reactions. Therapist interaction with patients occurs during the drug administration session to help the patient experience nervous system activation without fear or dissociation and foster adaptive regulation of challenging internal experiences. Non-drug administration sessions involved supportive psychotherapy that aimed to reconcile therapy experiences to facilitate insight and assist patients in planning for change in behaviors.

\section{Measures}

Demographics. Demographic measures assessed at intake included age, sex, gender identity, ethnicity, highest level of education, and current marital or partnership status.

History of traumatic experiences. The Life Event Checklist 5 (LEC-5) was used at intake to assess for exposure to potentially traumatic events across 17 domains (e.g., natural disaster, physical injury, sexual assault, war exposure, sudden violent death) (Gray et al., 2004). For each item, patients specified whether they directly experienced, witnessed, learned of it happening to a close family member or friend, or were exposed through work to the event. Total LEC scores were calculated by summing LEC item scores, and total scores range from 0 to 68 . To assess for traumatic experiences before age 18, the 10-item ACE scale was included at intake as a measure for childhood physical abuse, sexual abuse, financial insecurity, familial mental health illness, substance abuse, incarceration, and lack of emotional support (Felitti et al., 1998). Total ACE scores range from 0 to 10 , with scores 4 or more indicating severe childhood trauma burden.

Post-traumatic stress disorder. The Primary Care Post Traumatic Stress Disorder 5 (PC-PTSD-5) was used at intake and session six to assess PTSD symptoms. The PCPTSD-5 is a five-item dichotomous questionnaire that was developed as a measure to determine whether patients experience common symptoms of PTSD (Prins et al., 2016). Patients were identified with probable PTSD if scores were greater than 3 at intake and session six (Prins et al., 2016). To assess changes in PTSD symptom severity, the scale was modified to include an intensity rating wherein patients responded to each item on a scale of 0 ("not at all") to 4 ("extremely") for the past month. Total PTSD scores ranged from 0 to 20, with higher scores indicating higher symptom severity. Mean PTSD scores were calculated by finding the average item score with total scores ranging from 0 to 4 .

Depression. The Patient Health Questionnaire 2 (PHQ-2) was used at intake and session six to assess depression symptoms. The PHQ-2 is a two-item measure used to assess depressive symptoms (Kroenke, Spitzer, \& Williams, 2003). Each item was scored on a 4-point scale, examining the frequency of symptoms experienced from 0 ("not at all") to 3 
("nearly every day"). Patients in this study answered whether they felt anhedonia ("decreased interest or pleasure") or depressed mood ("down, depressed, or hopeless") in the past two weeks. Total scores ranged from 0 to 6 with higher scores indicating greater depressive symptoms. A score greater than or equal to 3 indicated likely major depression (Kroenke et al., 2003).

Anxiety. The Generalized Anxiety Disorder 2 (GAD-2) was used at intake and session six to assess anxiety symptoms. The GAD-2 is a two-item measure used to assess symptoms of generalized anxiety (Terrill et al., 2015). Each item was scored on a 4-point scale related to the frequency of the symptoms experienced ranging from 0 ("not at all") to 3 ("nearly every day"). In this study, patients answered whether they felt nervous, anxious, on edge or unable to control worrying in the past two weeks. Scores ranged from 0 to 6 , with higher scores indicating higher anxiety. A score greater than or equal to 3 identified clinically significant anxiety symptoms (Plummer et al., 2016).

Global disability. The Sheehan Disability Scale was used at intake and session six to assess the extent psychological symptoms may have on global functioning (Sheehan \& Sheehan, 2008). In this study, a three-item questionnaire assessed the impact of psychological symptoms on disability in academic or career, social, and familial or home functioning. Each item was scored on a 5-point Likert scale from 0 ("not at all) to 4 ("extremely"). Total scores ranged from 0 to 12 , with higher scores indicating a higher level of disability in global functioning.

\section{Data analysis}

Following a determination from the institutional review board at Ohio State University that this research was exempt from review, de-identified data were obtained from an outpatient mental health clinic located in Colorado and were retrospectively analyzed. Clinical records data included data that were collected from adult patients that initiated treatment between 2018 and 2020. Data was de-identified through an anonymized coding scheme (random patient number) that was entered into the clinical tracking software system (SurveyGizmo) by therapists at the clinic. Descriptive analyses of demographic and trauma history recorded at intake were calculated. Mean change scores on all symptoms and disability measures were calculated by subtracting scale scores at session six from scores provided at intake. Symptoms and disability outcomes were calculated by comparing mean differences in scale scores (e.g., PTSD, depression, anxiety, disability) at intake compared to session six by a series of paired samples $t$-tests. Effect size for each $t$-test was assessed by calculating Cohen's d statistics. Meaningful reductions in symptoms were interpreted when Cohen's d effect sizes were in the medium range $(d>0.30)$. Pairedsamples $t$-tests comparing mean symptom severity and disability scores (e.g., PTSD, depression, anxiety, disability) at intake with scores from session six among patients who received ketamine-assisted therapy while in the clinic.
Table 1. Demographic characteristics

\begin{tabular}{|c|c|}
\hline Characteristic $(N=18)$ & $\mathrm{M}(\mathrm{SD})$ or $\%$ \\
\hline Age & $45.22(12.90)$ \\
\hline \multicolumn{2}{|l|}{$\operatorname{Sex}$} \\
\hline Female & $55.60 \%$ \\
\hline Male & $44.40 \%$ \\
\hline \multicolumn{2}{|l|}{ Gender } \\
\hline Female & $55.60 \%$ \\
\hline Male & $38.90 \%$ \\
\hline Gender-fluid & $0 \%$ \\
\hline Other & $5.6 \%$ \\
\hline \multicolumn{2}{|l|}{ Race } \\
\hline Caucasian/White & $100 \%$ \\
\hline \multicolumn{2}{|l|}{ Are you Hispanic or Latino? } \\
\hline Yes & $5.60 \%$ \\
\hline No & $94.40 \%$ \\
\hline \multicolumn{2}{|l|}{ Highest education level } \\
\hline Some college credit, no degree & $22.20 \%$ \\
\hline Associate's degree & $11.10 \%$ \\
\hline Bachelor's Degree & $27.80 \%$ \\
\hline Master's Degree & $22.20 \%$ \\
\hline $\begin{array}{l}\text { Advanced professional or Doctoral } \\
\text { Degree (e.g., Ph.D., M.D., etc.) }\end{array}$ & $16.70 \%$ \\
\hline \multicolumn{2}{|l|}{ Marital or Partnership Status } \\
\hline Never married & $22.20 \%$ \\
\hline Married and living with spouse & $61.1 \%$ \\
\hline Living with partner & $5.60 \%$ \\
\hline Divorced or separated & $11.10 \%$ \\
\hline
\end{tabular}

Note. All demographic characteristics were assessed once at intake. Total percentages may not sum up to $100 \%$ due to rounding and the option to select more than one option of those provided.

Analyses were conducted using SPSS version 25 and 26 (IBM Corp, 2018, 2019).

\section{RESULTS}

\section{Participant characteristics}

As Table 1 reveals, the overall sample $(N=18)$ was comprised of middle-aged $\left(M_{\text {age }}=45.22, S D=12.90\right)$ Caucasian/white (100\%) men (56\%) and women (44\%). A large proportion of patients in the sample reported having a bachelor's degree $(28 \%)$ and were married and living with a spouse $(61 \%)$. With respect to trauma exposure, and as Table 2 shows, participants had significant childhood and adult trauma represented by their total ACE scores $(M=$ $3.17, \mathrm{SD}=2.15)$ and total life event checklist (LEC) scores $(M=11.33, \mathrm{SD}=7.38)$, respectively. Notably, there are high rates of personal experience with transportation accidents (50\%), physical assault (39\%), and unwanted or uncomfortable sexual experiences (56\%). In addition, a majority $(67 \%)$ of individuals reported having a household member that suffered from mental illness or attempted suicide in childhood. Most participants at intake had a probable diagnosis of PTSD (83\%) with moderate total PTSD symptom severity $(M=10.39, S D=6.04)$. On 
Table 2. Trauma inventory and scale scores of total sample at intake and among each subsample of those who completed intake, six sessions, and twelve sessions of therapy

\begin{tabular}{lr}
\hline Trauma Inventory \& Scale Scores & \\
& $\mathrm{a}=18$ \\
& $\mathrm{M}(\mathrm{SD})$ or $\%$ \\
\hline
\end{tabular}

Adverse Childhood Experience (ACE) ${ }^{\mathrm{b}}$

Did a parent or other adult in the $50 \%$

household often or very often...

Swear at you, insult you, put you

down, or humiliate you? or Act in a

way that made you afraid that you

might be physically hurt?

Did a parent or other adult in the

household often or very often... Push, grab, slap, or throw something at you? or Ever hit you so hard that you had marks or were injured?

Did an adult or person at least 5 years older than you ever... Touch or fondle you or have you touch their body in a sexual way? or Attempt or actually have oral, anal, or vaginal intercourse with you?

Did you often or very often feel that... No one in your family loved you or thought you were important or special? or Your family didn't look out for each other, feel close to each other, or support each other?

Did you often or very often feel that ... You didn't have enough to eat, had to wear dirty clothes, and had no one to protect you? or Your parents were too drunk or high to take care of you or take you to the doctor if you needed it?

Were your parents ever separated or divorced?

Was your mother or stepmother: Often or very often pushed, grabbed, slapped, or had something thrown at her? or Sometimes, often, or very often kicked, bitten, hit with a fist, or hit with something hard? or Ever repeatedly hit over at least a few minutes or threatened with a gun or knife?

Did you live with anyone who was a problem drinker or alcoholic, or who used street drugs?

Was a household member depressed or mentally ill, or did a household member attempt suicide?

Did a household member go to prison?

Total ACE Score

The Life Event Checklist (LEC)

Natural Disaster:

Event happened to me

Witnessed it

Learned about it

$33.30 \%$

$11.10 \%$

$22.20 \%$

Part of my job
Table 2. Continued

\begin{tabular}{cc}
\hline Trauma Inventory \& Scale Scores & \\
& \\
& $\mathrm{M}(\mathrm{SD})$ or $\%$ \\
\hline
\end{tabular}

Fire or explosion:

Event happened to me

Witnessed it

$0 \%$

Learned about it

$16.70 \%$

Part of my job

$22.20 \%$

$11.10 \%$

Transportation accident:

Event happened to me $\quad 50.00 \%$

Witnessed it $\quad 27.80 \%$

Learned about it $\quad 16.70 \%$

Part of my job

Serious accident at work, home, or during recreational activity:

Event happened to me $\quad 11.10 \%$

Witnessed it $22.20 \%$

Learned about it $\quad 33.30 \%$

Part of my job

Exposure to toxic substance:

Event happened to me

Witnessed it

Learned about it

Part of my job

Physical assault:

Event happened to me $\quad 38.90 \%$

Witnessed it $27.80 \%$

Learned about it $\quad 11.10 \%$

Part of my job $\quad 5.60 \%$

Assault with a weapon:
Event happened to me

Witnessed it $\quad 27.80 \%$

Learned about it $\quad 22.20 \%$

Part of my job $\quad 5.60 \%$

Sexual assault:

Event happened to me $\quad 33.30 \%$

Witnessed it $\quad 0 \%$

Learned about it $\quad 22.20 \%$

Part of my job $\quad 5.60 \%$

Other unwanted or uncomfortable sexual experience:

Event happened to me $\quad 55.60 \%$

Witnessed it $\quad 16.70 \%$

Learned about it $\quad 22.20 \%$

Part of my job $\quad 5.60 \%$

Combat or exposure to a war-zone:

Event happened to me $\quad 0 \%$

Witnessed it $\quad 0 \%$

Learned about it $\quad 22.20 \%$

Part of my job $\quad 0 \%$

Captivity:

Event happened to me $\quad 5.60 \%$

Witnessed it $\quad 0 \%$

Learned about it $\quad 5.60 \%$

Part of my job $\quad 5.60 \%$

Life-threatening illness or injury:

Witnessed it $\quad 55.60 \%$

Learned about it $\quad 22.20 \%$

Part of my job $\quad 5.60 \%$

(continued) 
Table 2. Continued

\begin{tabular}{|c|c|}
\hline Trauma Inventory \& Scale Scores ${ }^{\mathrm{a}}$ & $\begin{array}{c}N=18 \\
\mathrm{M}(\mathrm{SD}) \text { or } \%\end{array}$ \\
\hline \multicolumn{2}{|l|}{ Severe human suffering: } \\
\hline Event happened to me & $11.10 \%$ \\
\hline Witnessed it & $22.20 \%$ \\
\hline Learned about it & $16.70 \%$ \\
\hline Part of my job & $16.70 \%$ \\
\hline \multicolumn{2}{|l|}{ Sudden violent death: } \\
\hline Event happened to me & $11.1 \%$ \\
\hline Witnessed it & $5.60 \%$ \\
\hline Learned about it & $38.90 \%$ \\
\hline Part of my job & $16.70 \%$ \\
\hline \multicolumn{2}{|l|}{ Sudden accidental death: } \\
\hline Event happened to me & $11.10 \%$ \\
\hline Witnessed it & $5.60 \%$ \\
\hline Learned about it & $38.90 \%$ \\
\hline Part of my job & $16.70 \%$ \\
\hline \multicolumn{2}{|l|}{$\begin{array}{l}\text { Serious injury, harm, or death you } \\
\text { caused to someone else: }\end{array}$} \\
\hline Event happened to me & $11.10 \%$ \\
\hline Witnessed it & $0 \%$ \\
\hline Learned about it & $0 \%$ \\
\hline Part of my job & $11.10 \%$ \\
\hline \multicolumn{2}{|l|}{$\begin{array}{l}\text { Any other very stressful event or } \\
\text { experience: }\end{array}$} \\
\hline Event happened to me & $77.80 \%$ \\
\hline Witnessed it & $27.80 \%$ \\
\hline Learned about it & $16.70 \%$ \\
\hline Part of my job & $22.20 \%$ \\
\hline Total LEC & $11.33(7.38)$ \\
\hline
\end{tabular}

Note. Total scale scores represent symptom severity assessed at baseline.

${ }^{\text {a }}$ Scale score interpretation: Total ACE (range 0-10, higher scores indicate greater childhood trauma exposure); Total LEC (range 068 , higher scores indicate greater trauma exposure across the lifespan).

${ }^{\mathrm{b}}$ Percentages reported in ACE questionnaire were of respondents who answered 'yes' to the item asked.

average, the sample at intake met the criteria for clinically significant depression $(M=3.89, S D=2.29)$ and anxiety $(M=3.67, S D 2.14)$. They also demonstrated severe global disability in functioning $(M=8.24, S D=3.70)$.

\section{Symptom improvement after six sessions of ketamine- assisted therapy}

As evidenced in Table 3, patients that completed six sessions of therapy reported meaningful improvement (e.g., medium effect size) in total PTSD symptoms $(P=0.058 ; d=-0.48)$ and global disability in functioning $(P=0.050 ; d=-0.52)$. They also exhibited statistically significant reductions in severity of depression symptoms $(P=0.019 ; d=-0.61)$. However, there was no significant change in anxiety symptoms $(P=0.249 ; d=-0.28)$.

\section{DISCUSSION}

Although recent evidence suggests that ketamine-assisted therapy using sublingual and intramuscular ketamine is efficacious in reducing depression and anxiety symptoms (Dore et al., 2019), no study to date has examined the use of sublingual ketamine as an adjunct to somatic-focussed psychotherapy among trauma-exposed individuals in a realworld clinic setting. This study is the first of its kind to examine prospectively collected clinical chart data from an outpatient clinic in Colorado providing this specific somatic therapy in a trauma-exposed population. Findings from the present study showed that, among patients with probable PTSD, there were meaningful, though not statistically significant, reductions in total intensity of PTSD symptoms and improvement in global life functioning, as well as statistically significant and meaningful improvements in depression symptoms over the course of six sessions of therapy.

The findings from the present study are consistent with the literature showing improvement in traumatic stress and depression symptoms following evidenced-based psychotherapeutic interventions (Brom et al., 2017; Bisson et al., 2007, 2013), and improvements among patients receiving sublingual and intramuscular ketamine in a clinic setting (Dore et al., 2019). However, findings from our study extend these prior studies by showing that sublingual ketamine as an augmentation to somatic psychotherapy provided in a realworld clinic setting has the potential to meaningfully reduce PTSD and depression symptoms among a trauma-exposed population. Although these initial findings are promising,

Table 3. Change in Symptom Severity of Patients Between Intake and Sixth Session of Therapy ( $t$-test)

\begin{tabular}{|c|c|c|c|c|c|c|c|}
\hline Measure $(\mathrm{N})^{\mathrm{a}}$ & $\begin{array}{l}\text { Intake } \\
\text { M (SD) }\end{array}$ & $\begin{array}{l}\text { Six sessions } \\
M(\mathrm{SD})\end{array}$ & $\begin{array}{c}\text { Change score } \\
M(\mathrm{SD})\end{array}$ & $t$-test & $P$ & $95 \% \mathrm{CI}$ & Effect size (Cohen's d) \\
\hline Total PTSD (18) & $10.39(6.04)$ & $8.44(4.76)$ & $-1.94(4.07)$ & 2.03 & 0.058 & {$[-0.02,0.96]$} & -0.48 \\
\hline PHQ-2 (18) & $3.88(2.30)$ & $2.72(2.44)$ & $-1.17(1.91)$ & 2.58 & $0.019^{*}$ & {$[0.09,1.11]$} & -0.61 \\
\hline GAD-2 (18) & $3.66(2.14)$ & $3.06(2.13)$ & $-0.61(2.17)$ & 1.19 & 0.249 & {$[-0.19,0.75]$} & -0.28 \\
\hline SDS (17) & $8.24(3.70)$ & $6.41(4.15)$ & $-1.82(3.54)$ & 2.12 & 0.050 & {$[0.001,1.02]$} & -0.52 \\
\hline
\end{tabular}

Note. This table compares symptom severity in patients that had ketamine-assisted psychotherapy and completed six sessions of therapy $(N$ $=20$ ). N's may vary between measures due to missing survey data.

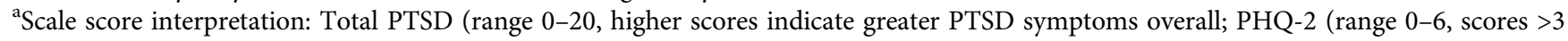
indicate clinically significant depression); GAD-2 (range 0-6, scores $>3$ indicate clinically significant anxiety); SDS (range 0-12, higher scores indicate greater global functional impairment).

${ }^{\star} P<0.05,{ }^{* \star} P<0.01,{ }^{* *} P<0.001$. 
there has yet been no randomized controlled trial testing the efficacy of sublingual ketamine-assisted therapy for patients with PTSD. Furthermore, this study was underpowered and not representative of the population of patients receiving sublingual ketamine assisted somatic therapy. For example, although the statistical tests revealed only a marginal reduction in PTSD symptoms $(P=0.058)$, the effect size for this finding was medium $(d=-0.48)$ suggesting that a fully powered study might have revealed conventionally defined statistical significance. Similarly, improvements in depression and global disability in functioning, also with moderate effect sizes ( $d=-0.61$ and -0.52 , respectively), further support that these findings are a meaningful reflection of the improvements reported by these patients. Nevertheless, more research is needed to determine the clinical safety of this approach and demonstrate efficacy when comparing against a control group in larger patient samples.

This study has several limitations. First, as a clinic chart review study, we are limited to including those patients who choose to attend treatment at this specific clinic and the subset of those who stay in treatment over time, which may have led to an overestimation of response as those who stayed in treatment could be those more likely to improve. Second, expectancy effects were not assessed in this study, which could explain the response to this treatment. Furthermore, the study lacks any independent or clinicianrated diagnostic data or clinical response data, relying solely on patients' self-report which could have overestimated the extent of positive outcomes due to social desirability. Third, the clinic used modified symptom screening measures (e.g., PCPTSD5) to track clinical outcomes, which were not developed to be used to track symptoms improvement. Although it is possible that self-report screening tools are sensitive to symptom changes in the same way as clinician administered batteries of diagnostic measures or other selfreport measures, a full examination of these screening tools is needed before conclusions can be made about their utility as ongoing symptom tracking assessments.

Fourth, the clinic uses a psychotherapeutic approach that incorporated somatic-focused processes and it is unknown to what extent the psychotherapeutic approach accounts for the decreases in mental health symptoms and improvement in functioning. Although the quantitative data from this study established a signal of the utility of this approach, a qualitative study would better help elucidate the personal experiences of this treatment and thus should be explored in future research. Fifth, there is variability in dosing and bioavailability of ketamine in patients at this clinic, which could account for differences in outcomes across patients in this study or in comparing findings from this study to other published research. For example, the clinic in this study used varying doses between 100 and $200 \mathrm{mg}$ of ketamine and instructed patient to hold the lozenge under the tongue as long as they could or until it dissolved. But there was no verification of procedure or measure of time that elapsed before starting psychotherapy and this could have confounded the results. Finally, data from this study was from one clinic in Colorado which limits generalizability to the broader population of patients that might be receiving this type of therapy in other places in the US.

This study was the first of its kind to assess the utility of sublingual ketamine assisted somatic therapy among patients with a history of trauma exposure. That a variety of symptom domains (PTSD, depression) were meaningfully and/or statistically significantly decreased in a relatively short amount of time (six sessions) suggests that this intervention could be an important step in the innovation of treatment options for this population, but further understanding of the efficacy of this treatment approach, its mechanisms of action, and the safety and tolerability in diverse patient samples is needed. Although this study is limited in scope and generalizability, the findings highlight the importance of conducting rigorously controlled trials to examine the potential therapeutic efficacy of sublingual ketamine as an adjunct to psychotherapy for people with trauma-based mental health problems.

Financial support: Funding for the study was provided by Innate Path, which was the clinic that collected the chart review data that was used in this study. The funder was not involved in the analysis of data, the decision to publish, or preparation of the manuscript.

Declaration of interests: Dr. Davis is a board member of Source Research Foundation and Lead Trainer at Fluence. These organizations were not involved in the design/ execution of this study or the interpretation or communication of findings.

\section{REFERENCES}

Allem, J. P., Soto, D. W., Baezconde-Garbanati, L., et al. (2015). Adverse childhood experiences and substance use among Hispanic emerging adults in Southern California. Addictive Behaviors, 50, 199-204.

Bader, K., Schafer, V., Schenkel, M., et al. (2007). Adverse childhood experiences associated with sleep in primary insomnia. Journal of Sleep Research, 16(3), 285-296.

Bisson, J. I., Ehlers, A., Matthews, R., et al. (2007). Psychological treatments for chronic post-traumatic stress disorder. Systematic review and meta-analysis. The British Journal of Psychiatry, 190, 97-104.

Bisson, J. I., Roberts, N. P., Andrew, M., et al. (2013). Psychological therapies for chronic post-traumatic stress disorder (PTSD) in adults. The Cochrane Database of Systematic Reviews, Epub ahead of print 2013/12/18. https://doi.org/10.1002/14651858. CD003388.pub4.(12):Cd003388.

Brady, K., Pearlstein, T., Asnis, G. M., et al. (2000). Efficacy and safety of sertraline treatment of posttraumatic stress disorder: A randomized controlled trial. JAMA, 283(14), 1837-1844.

Brady, F., Warnock-Parkes, E., Barker, C., et al. (2015). Early insession predictors of response to trauma-focused cognitive therapy for posttraumatic stress disorder. Behaviour Research and Therapy, 75, 40-47. 
Brom, D., Stokar, Y., Lawi, C., et al. (2017). Somatic experiencing for posttraumatic stress disorder: A randomized controlled outcome study. Journal of Traumatic Stress, 30(3), 304-312.

Chilcoat, H. D., \& Breslau, N. (1998). Posttraumatic stress disorder and drug disorders: Testing causal pathways. Archives of General Psychiatry, 55(10), 913-917.

Crouch, E., Probst, J. C., Radcliff, E., et al. (2019). Prevalence of adverse childhood experiences (ACEs) among US children. Child Abuse \& Neglect, 92, 209-218.

D'Andrea, D., \& Andrew Sewell, R. (2013). Transient resolution of treatment-resistant posttraumatic stress disorder following ketamine infusion. Biological Psychiatry, 74(9), e13-e14.

Dore, J., Turnipseed, B., Dwyer, S., et al. (2019). Ketamine assisted psychotherapy (KAP): Patient demographics, clinical data and outcomes in three large practices administering ketamine with psychotherapy. Journal of Psychoactive Drugs, 51(2), 189-198.

Feder, A., Costi, S., Rutter, S. B., Collins, A. B., Govindarajulu, U., Jha, M. K., et al. (2021). A randomized controlled trial of repeated ketamine administration for chronic posttraumatic stress disorder. American Journal of Psychiatry, 178(2), 193-202.

Feder, A., Parides, M. K., Murrough, J. W., et al. (2014). Efficacy of intravenous ketamine for treatment of chronic posttraumatic stress disorder: A randomized clinical trial. JAMA Psychiatry, 71(6), 681-688.

Felitti, V. J., Anda, R. F., Nordenberg, D., et al. (1998). Relationship of childhood abuse and household dysfunction to many of the leading causes of death in adults. The Adverse Childhood Experiences (ACE) Study. American Journal of Preventive Medicine, 14(4), 245-258.

Friedman, M. J., Marmar, C. R., Baker, D. G., et al. (2007). Randomized, double-blind comparison of sertraline and placebo for posttraumatic stress disorder in a Department of Veterans Affairs setting. The Journal of Clinical Psychiatry, 68(5), 711720.

Glue, P., Neehoff, S. M., Medlicott, N. J., et al. (2018). Safety and efficacy of maintenance ketamine treatment in patients with treatment-refractory generalised anxiety and social anxiety disorders. Journal of Psychopharmacology, 32(6), 663-667.

Gray, M. J., Litz, B. T., Hsu, J. L., et al. (2004). Psychometric properties of the life events checklist. Assessment, 11(4), 330341.

Hall Brown, T. S., Akeeb, A., \& Mellman, T. A. (2015). The role of trauma type in the risk for insomnia. Journal of Clinical Sleep Medicine, 11(7), 735-739.

Hughes, K., Bellis, M. A., Sethi, D., et al. (2019). Adverse childhood experiences, childhood relationships and associated substance use and mental health in young Europeans. European Journal of Public Health, 29(4), 741-747.

IBM Corp. (2018). IBM SPSS statistics for windows. Armonk, NY: IBM Corp.

IBM Corp. (2019). IBM SPSS statistics for windows. Armonk, NY: IBM Corp.

Imel, Z. E., Laska, K., Jakupcak, M., et al. (2013). Meta-analysis of dropout in treatments for posttraumatic stress disorder. Journal of Consulting and Clinical Psychology, 81(3), 394-404.

Kessler, R. C. (2000). Posttraumatic stress disorder: The burden to the individual and to society. The Journal of Clinical Psychiatry, 61(Suppl. 5), 4-12 discussion 13-14.
Kessler, R. C., Sonnega, A., Bromet, E., et al. (1995). Posttraumatic stress disorder in the national comorbidity survey. Archives of General Psychiatry, 52(12), 1048-1060.

Koenen, K. C., Ratanatharathorn, A., Ng, L., et al. (2017). Posttraumatic stress disorder in the world mental health surveys. Psychological Medicine, 47(13), 2260-2274.

Kroenke, K., Spitzer, R. L., \& Williams, J. B. (2003). The patient health questionnaire-2: Validity of a two-item depression screener. Medical Care, 41(11), 1284-1292.

Maercker, A., Michael, T., Fehm, L., et al. (2004). Age of traumatisation as a predictor of post-traumatic stress disorder or major depression in young women. The British Journal of Psychiatry, 184, 482-487.

Maher, M. J., Rego, S. A., \& Asnis, G. M. (2006). Sleep disturbances in patients with post-traumatic stress disorder: Epidemiology, impact and approaches to management. CNS Drugs, 20(7), 567-590.

McGhee, L. L., Maani, C. V., Garza, T. H., et al. (2008). The correlation between ketamine and posttraumatic stress disorder in burned service members. Journal of Trauma, 64(2 Suppl), S195-S198, Discussion S197-S198.

Memon, R. I., Naveed, S., Faquih, A. E., Fida, A., Abbas, N., Chaudhary, A. M. D., \& Qayyum, Z. (2020). Effectiveness and safety of ketamine for unipolar depression: A systematic review. Psychiatric Quarterly, 91(4), 1147-1192.

Milanak, M. E., Gros, D. F., Magruder, K. M., et al. (2013). Prevalence and features of generalized anxiety disorder in Department of Veteran Affairs primary care settings. Psychiatry Research, 209(2), 173-179.

Mitchell, J. M., Bogenschutz, M., Lilienstein, A., Harrison, C., Kleiman, S., Parker-Guilbert, K., et al. (2021). MDMA-assisted therapy for severe PTSD: A randomized, double-blind, placebocontrolled phase 3 study. Nature Medicine, 27(6), 1025-1033.

Neylan, T. C., Marmar, C. R., Metzler, T. J., et al. (1998). Sleep disturbances in the Vietnam generation: Findings from a nationally representative sample of male Vietnam veterans. The American Journal of Psychiatry, 155(7), 929-933.

Panahi, Y., Moghaddam, B. R., Sahebkar, A., et al. (2011). A randomized, double-blind, placebo-controlled trial on the efficacy and tolerability of sertraline in Iranian veterans with post-traumatic stress disorder. Psychological Medicine, 41(10), 2159-2166.

Pietrzak, R. H., Goldstein, R. B., Southwick, S. M., et al. (2012). Psychiatric comorbidity of full and partial posttraumatic stress disorder among older adults in the United States: Results from wave 2 of the national epidemiologic survey on alcohol and related conditions. The American Journal of Geriatric Psychiatry, 20(5), 380-390.

Plummer, F., Manea, L., Trepel, D., et al. (2016). Screening for anxiety disorders with the GAD-7 and GAD-2: A systematic review and diagnostic metaanalysis. General Hospital Psychiatry, 39, 24-31.

Prins, A., Bovin, M. J., Smolenski, D. J., et al. (2016). The primary care PTSD screen for DSM-5 (PC-PTSD-5): Development and evaluation within a veteran primary care sample. Journal of General Internal Medicine, 31(10), 1206-1211.

Rodriguez, P., Holowka, D. W., \& Marx, B. P. (2012). Assessment of posttraumatic stress disorder-related functional impairment: A review. Journal of Rehabilitation Research and Development, 49(5), 649-665. 
Rosenblat, J. D., Carvalho, A. F., Li, M., et al. (2019). Oral ketamine for depression: A systematic review. The Journal of Clinical Psychiatry, 80(3).

Shadli, S. M., Kawe, T., Martin, D., et al. (2018). Ketamine effects on EEG during therapy of treatment-resistant generalized anxiety and social anxiety. The International Journal of Neuropsychopharmacology, Epub ahead of print 2018/05/03. https:// doi.org/10.1093/ijnp/pyy032.

Sheehan, K. H., \& Sheehan, D. V. (2008). Assessing treatment effects in clinical trials with the discan metric of the Sheehan Disability Scale. International Clinical Psychopharmacology, 23(2), 70-83.

Stein, M. B., Walker, J. R., Anderson, G., et al. (1996). Childhood physical and sexual abuse in patients with anxiety disorders and in a community sample. The American Journal of Psychiatry, 153(2), 275-277.

Terrill, A. L., Hartoonian, N., Beier, M., et al. (2015). The 7-item generalized anxiety disorder scale as a tool for measuring generalized anxiety in multiple sclerosis. International Journal of MS Care, 17(2), 49-56.

van der Kolk, B. A., Stone, L., West, J., et al. (2014). Yoga as an adjunctive treatment for posttraumatic stress disorder: A randomized controlled trial. The Journal of Clinical Psychiatry, 75(6), e559-e565.

Warner, C. H., Warner, C. M., Appenzeller, G. N., et al. (2013). Identifying and managing posttraumatic stress disorder. American Family Physician, 88(12), 827-834.

Watkins, L. E., Sprang, K. R., \& Rothbaum, B. O. (2018). Treating PTSD: A review of evidence-based psychotherapy interventions. Frontiers in Behavioral Neuroscience, 12, 258-258.

Westphal, M., Olfson, M., Gameroff, M. J., et al. (2011). Functional impairment in adults with past posttraumatic stress disorder: Findings from primary care. Depression and Anxiety, 28(8), 686-695. 